\title{
Upside-Down Rotation of a Breast Implant with Double Capsule Formation after Aesthetic Breast Augmentation: A Case Report
}

\author{
Wang Seok Lee, Sang Gue Kang \\ Department of Plastic and Reconstructive \\ Surgery, Soonchunhyang Seoul Hospital, \\ Soonchunhyang University College of \\ Medicine, Seoul, Korea
}

Some complications can occur after augmentation mammoplasty with a prosthesis. Double capsule formation is an uncommon complication, and it is especially rare for it to be accompanied by malrotation of a round implant. We report herein a case of double capsule formation with upside-down rotation of the implant after augmentation mammoplasty.

Keywords Biofilms, Breast, Breast implants, Mammaplasty, Rotation
This work was supported by the Soonchunhyang University Research Fund.

No potential conflict of interest relevant to this article was reported. computed tomographic imaging with contrast, we performed a change of the implant. A review of the clinical, radiological, and gross findings in this case is presented below.

\section{CASE REPORT}

A 27-year-old woman visited us complaining of contour deformity on the left breast after augmentation mammoplasty. The symptom was found during a physical exam at our clinic, and there was no history of any traumatic event affecting the breast. She had undergone bariatric surgery 2 years ago, and therefore also complained of excessive skin on the trunk and both thighs. She had a 2-year history of polycystic ovarian syndrome that was controlled well with oral contraceptives. The patient had undergone augmentation mammoplasty with round implants (Allergan [Allergan Inc., Irvine, CA, USA], type 110, $270 \mathrm{~mL}$ ) 1 month previously. In each breast, an inframammary fold incision was made and the prosthesis was placed in the subglandular pocket. There were no immediate complications. Upon physical examination, the implant in the left breast was palpable on the medial side of the breast. There was no bruising, erythema, tenderness, or signs of inflammation on the covering skin (Fig. 1). We also were not able to observe rippling of the implant. A moderate degree of breast asymmetry was shown. For further evaluation, chest computed tomography with contrast enhancement was carried out. Magnetic resonance imaging has the highest sensitivity and specificity for detecting implant rupture;
Received: Apr 14, 2018 Revised: May 9, 2018 Accepted: May 21, 2018 Correspondence: Sang Gue Kang Department of Plastic and Reconstructive Surgery, Soonchunhyang Seoul Hospital, Soonchunhyang University College of Medicine, 59 Daesagwan-ro, Yongsan-gu, Seoul 04401, Korea.

E-mail: sgkang@schmc.ac.kr

Copyright () 2018 The Korean Society for Aesthetic Plastic Surgery.

This is an Open Access article distributed under the terms of the Creative Commons At tribution Non-Commercial License (http://creativecommons.org/licenses/by-nc/4.0/) which permits unrestricted non-commercial use, distribution, and reproduction in any medium, provided the original work is properly cited. www.e-aaps.org 


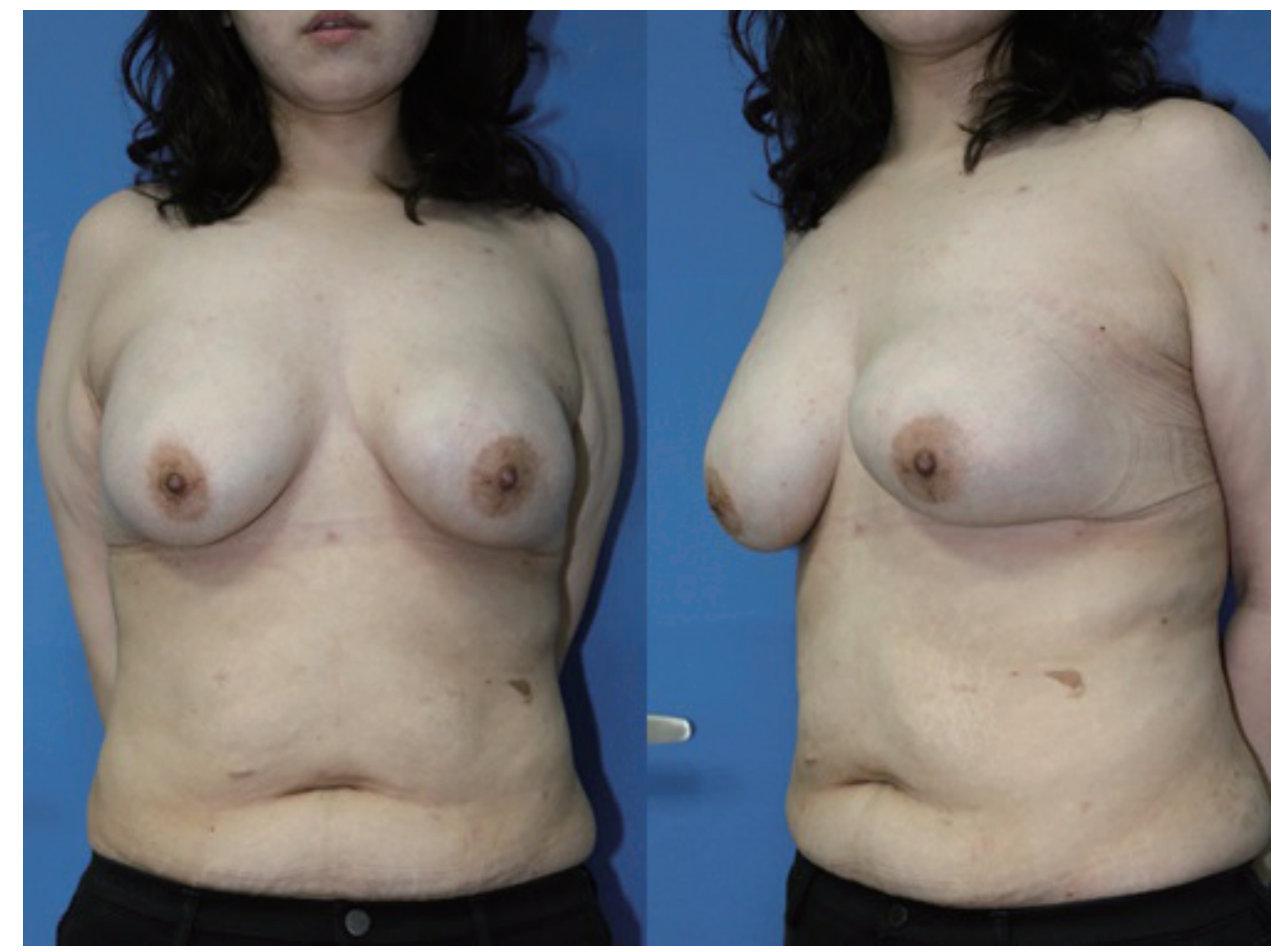

Fig. 1. Preoperative photographs. The preoperative photograph shows the bulged medial side of the left breast because of malrotation of the implant.

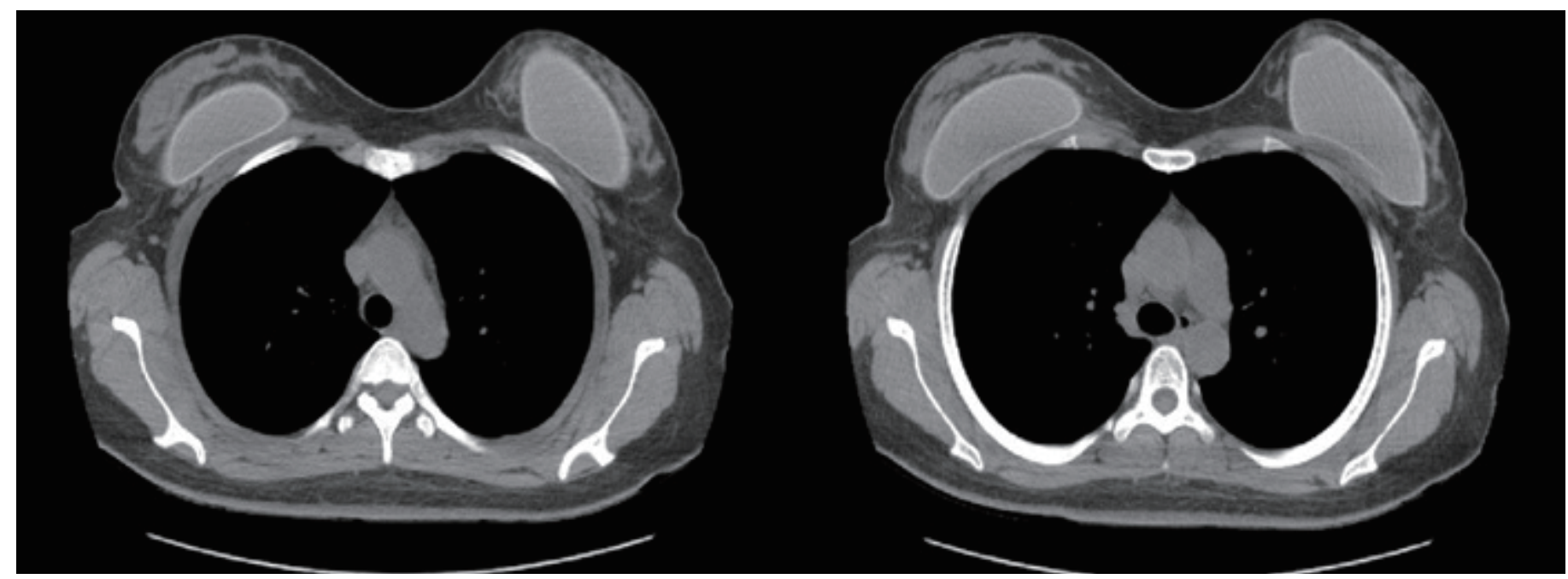

Fig. 2. Chest computed tomography imaging. Upside-down rotation of the implant on the left side of breast is shown.

however, our patient, as a cosmetic surgery patient, declined this imaging modality for financial reasons. Thus, computed tomography was used. There was no abnormal surrounding fluid collection or capsular rupture in the breast parenchyma, but upside-down rotation (along the vertical axis) of the silicone implant was observed on the left side of the breast (Fig. 2). We planned an implant exchange, in which the rotated implant would be removed and re- placed with another implant (same size, same type) via the original incision under local anesthesia. When the breast capsule was opened, the floor surface of the implant faced the breast tissue, not the chest wall, and almost half of the implant was covered by another intact capsule (Fig. 3). The capsule was intact and adherent on one side of the implant. Since the implant pocket was clean and healthy and the other breast had no problem with the inserted prosthesis, we 


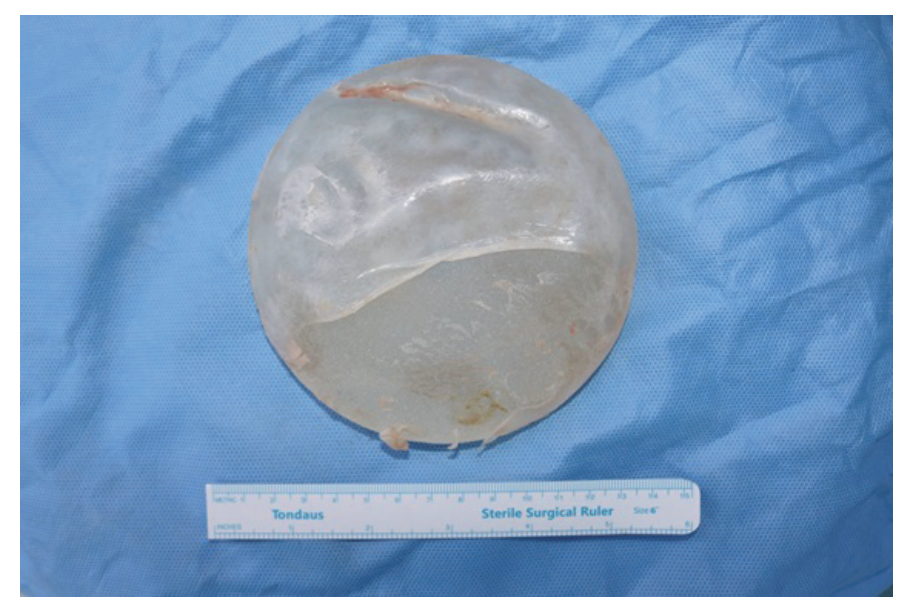

Fig. 3. Intraoperative findings. The implant was covered by a dense fibrous capsule.

decided to use the same implant and the same plane for symmetry. The pocket was irrigated and a new implant was inserted in the same pocket. The operation was finished after subcutaneous and cutaneous repair and aseptic compressive dressing without drainage. The patient used the dressing and there was no problem with the contour of her breast at 7 months of follow-up.

\section{DISCUSSION}

Augmentation mammoplasty with prosthesis implantation may cause several early and late complications. Among the various complications that can occur, double capsule formation is a well-known problem in breast augmentation. Even though the incidence of double capsule formation has not been reported, it is still a very rare complication. A double capsule refers to the clinical presentation of an encapsulated breast implant within another well-formed capsule. This phenomenon was first reported in 2002 by Pandya and Dickson [3]. In that article, the author explained the process of double capsule formation as involving shearing forces. Any vigorous movement of the implant and the adherent capsule can produce a shearing force between the breast tissue and the capsule-implant complex, leading to separation of the capsule-implant complex from the breast tissue, resulting in a hematoma with possible subsequent organization [1-4].

Matteucci and Fourie le [5] described the relationship between double capsule formation and dynamic malrotation. The author focused on the common history of minor trauma in people who experience double capsule and/or implant malrotation. He proposed that the implants initially integrate well, but following minor trauma, the interface between the cavity and the implant is disrupted. A new capsule then forms around the first capsule, producing a very-low-friction interface between the two, and rotation of the implant may occur [5].
There are 2 forces that act on the implant: gravity and compression by the pectoralis muscle, both of which act vertically downward and are applied to the center of gravity. These forces must be balanced by the resistance of the inferior margin of the pocket. As a result, the reaction force acts along the rib margin (i.e., along the posterior aspect of the prosthesis). Therefore, the active and the reactive forces act in parallel directions, generating coupled forces. Anomalous or excessive movements and external pressures can enhance the rotational moments [6].

Our patient had undergone massive weight loss, so her breast envelope was very movable and less elastic. Even though we performed a precise dissection to create the pocket, the pocket might have been more unstable than in other patients with more elastic skin. Moreover, when the patient changed her position, the vector of the forces made by the breast tissue also changed. This worsened the instability of the pocket and the implant's position. Many studies have investigated breast augmentation in patients who have undergone massive weight loss. A high rate of implant malposition (up to $60 \%$ ) has been reported; however, no study to date has exclusively focused on this patient population to educate surgeons and patients about how long after weight loss these complications tend to occur, and what the overall risk may be [7]. A surgeon who consults with a patient who has experienced massive weight loss should note the unique characteristics of the patient, and should carry out comprehensive surgical planning to obtain an optimal result. Future prospective and larger studies are needed to develop more helpful guidelines.

\section{PATIENT CONSENT}

Patient provided written consent for the use of her images.

\section{REFERENCES}

1. Baeke JL. Breast deformity caused by anatomical or teardrop implant rotation. Plast Reconstr Surg 2002;109:2555-64.

2. Irkoren S, Sivrioglu N. Can excessive breastfeeding be the cause for the upside-down rotation of a breast implant? A case report. Breastfeed Med 2013;8:515-6.

3. Pandya AN, Dickson MG. Capsule within a capsule: an unusual entity. Br J Plast Surg 2002;55:455-6.

4. Hall-Findlay EJ. Breast implant complication review: double capsules and late seromas. Plast Reconstr Surg 2011;127:56-66.

5. Matteucci P, Fourie le R. Double capsules related to dynamic malrotation of breast implants: a causal link? Br J Plast Surg 2004;57:289.

6. Panettiere P, Marchetti L, Accorsi D. Rotation of anatomic prostheses: a possible cause of breast deformity. Aesthetic Plast Surg 2004;28:348-53.

7. Coombs DM, Srivastava U, Amar D, et al. The challenges of augmentation mastopexy in the massive weight loss patient: technical considerations. Plast Reconstr Surg 2017;139:1090-9. 